\title{
SOP 13: Pharmacokinetic Data Analysis
}

\section{Background and Objectives}

Pharmacokinetic studies are an integrated part of the development program of a new drug. They are intended to define the time course of drug and major metabolite concentrations in plasma and other biological fluids in order to obtain information on absorption, distribution, metabolism, and elimination.

In preclinical studies, pharmacokinetics $(\mathrm{PK})$ is often used to interpret toxicological results. Animal toxicokinetic data can be used to guide dosage selection and escalation schemes in phase I dose tolerance studies. Pharmacokinetically guided dose escalation (PGDE) schemes are an example of the use of preclinical pharmacokinetic data for clinical dose finding in phase I studies.

In clinical studies, PK is mainly required for dose finding and dose escalation studies. In phase II and phase III studies, the randomized concentration-controlled clinical trial should be regarded as alternative to the traditional dosecontrolled trial, particularly if interindividual variability of pharmacokinetic parameters is large. Population pharmacokinetic data analysis may reveal the influence of patientspecific factors on pharmacokinetic parameters providing valuable information for dosage individualization.

For certain drugs, particularly those with a narrow therapeutic range, $\mathrm{PK}$ is used in clinical routine to adapt drug dosage to the individual patient (therapeutic drug monitoring).

The statistical evaluation problem of pharmacokinetic data is defined by the tasks to describe the time course of the amount or concentration of the drug in the body and its compartments and to determine rate constants by which the drug reaches the target organs/tissues, by which the drug is activated or bound and by which it is eliminated from the body.

\section{Relevant Pharmacokinetic Parameters}

The following pharmacokinetic parameters should be determined for each new drug:
- Total clearance (CL),

- Fraction of dose excreted unchanged in urine $\left(\mathrm{f}_{\mathrm{e}}\right)$,

- Volume of distribution at steady state $\left(\mathrm{V}_{\mathrm{ss}}\right)$,

- Volume of distribution during the terminal phase $\left(\mathrm{V}_{\mathrm{Z}}\right)$,

- Blood/plasma concentration ratio,

- Terminal half-life $\left(\mathrm{t}_{1 / 2} \mathrm{z}\right)$,

- Fraction of unbound drug in plasma $\left(f_{u}\right)$,

- Bioavailable fraction of dose (F), if applicable, and

- Absorption rate constant $\left(\mathrm{k}_{\mathrm{a}}\right)$, if applicable.

For each dosage regimen the following parameters should be known as well:

- Area under the plasma concentration time curve (AUC),

- Maximum concentration $\left(\mathrm{C}_{\max }\right)$,

- Minimum concentration $\left(\mathrm{C}_{\min }\right)$ after repeated dosing, and

- Time of $\mathrm{C}_{\max }\left(\mathrm{t}_{\max }\right)$.

In addition, the effective and toxic concentrations should be assessed.

\section{Study Considerations}

In order to generate reliable pharmacokinetic data, special care has to be taken regarding study design, sample collection, and processing.

\section{Study Design}

The determinants of a study design are:

- Route, mode and schedule of drug administration, and - Sites, methods and time points of sampling.

The number of subjects to be examined and the number of samples to be taken from each subject should be planned in advance on the basis of appropriate statistical considerations.

\section{Blood Sampling}

Blood should be sampled at predetermined time points by vein puncture or through an indwelling cannula. If the drug was administered intravenously, sampling should be

\section{KARGER}

Fax +497614520714

E-mail Information@Karger.de

www.karger.com
(C) 2003 S. Karger GmbH, Freiburg 
performed in the arm opposite to that used for drug administration to avoid contamination. If possible, $1 \mathrm{ml}$ blood should be withdrawn through the cannula prior to sampling and discarded. The exact collection time of each blood sample must be documented in a Case Report Form in conjunction with any irregularity or reasons for deviations. Samples should be filled in tubes containing an adequate anticoagulant and centrifuged immediately for separation of plasma under standardized conditions. It should be clarified that the anticoagulant does not interfere with the drug or its metabolites.

\section{Urine Collection}

Urine is collected in adequate containers according to the predetermined sampling intervals. Appropriate measures have to be taken in order to guarantee complete urine collection within a sampling interval. Urine volume and $\mathrm{pH}$ should be determined immediately after the end of a sampling interval.

\section{Sample Processing and Transport}

Samples should be divided into aliquots, if possible, frozen immediately and stored at an appropriate temperature. Samples should be shipped to the laboratory on dry ice and kept frozen until analysis.

\section{Data Handling}

\section{Data Entry}

Spread sheet interfaces (e.g. MS Excel or Lotus 1.2.3) connected with databases (e.g. MS Access, dBASE) facilitate a straightforward data entry and are helpful in solitary evaluations. For routine applications and comprehensive drug development programs, interfaces to integrated preclinical and clinical databases are more efficient. It is recommended to separate primary data and modeling software to avoid uncontrolled data modifications.

\section{Data Editing}

Data editing should include a set of procedures for detecting and correcting errors. Criteria for declaring data usable or unusable (e.g. time of blood sampling missing, concentrations with missing dosing information) should be predefined in the study protocol or in a study/project-specific SOP.

\section{Outliers}

Raw data should be carefully examined to determine if there are values not fitted well by the model (outliers). The reason for declaring a data point to be an outlier should preferably be specified prospectively in the study protocol. Physiological or study-related events that led to the exclusion of an outlier should be explained in the data report (see below 'Pharmacokinetic Report'). Outliers should be excluded from data analysis, particularly if they have a disproportionate effect on estimated parameters.

\section{Noncompartmental Data Analysis}

Noncompartmental analysis (NCA) does not require the assumption of a specific compartmental model for the drug of interest. However, a relatively large number of data points is required for a reliable estimation of pharmacokinetic parameters.

\section{Estimation of Terminal Elimination Rate Constant}

A minimum requirement is to have 3-4 observations in the terminal slope. By means of log-linear regression on those observations, the terminal elimination rate constant $\left(\lambda_{\mathrm{z}}\right)$ can be estimated.

\section{Estimation of $A U C$ and $A U M C$}

The AUC and the area under the first moment curve (AUMC) can be estimated by means of the linear trapezoidal rule or by the log-linear trapezoidal rule. In general, the linear trapezoidal rule should be applied for increasing or equal concentrations and the log-linear trapezoidal rule for decreasing concentrations.

For calculating AUC and AUMC from zero to infinity, the extrapolated area from the last sampling time to infinity has to be added to the sum of individual trapezoidal areas. The extrapolated area should not exceed $20-25 \%$ of the total area.

\section{Calculation of Pharmacokinetic Parameters}

Using the primary parameters $\lambda_{\mathrm{z}}$, AUC and AUMC, the following pharmacokinetic parameters can be calculated according to standard equations: $\mathrm{CL}, \mathrm{V}_{\mathrm{Z}}, \mathrm{V}_{\mathrm{ss}}$, and $\mathrm{t}_{1 / 2} \mathrm{Z}$.

\section{Compartmental Data Analysis}

Compartment models have served for a long time as paradigm of pharmacokinetic modeling. Program systems have been designed either as compartment model library or as compartment model generator where the user defines the model. Dosing schedules are translated to input functions depending on the site of administration (e.g. perorally, intraperitoneally, intravenously), the duration of administration (e.g. bolus, continuous infusion), or delays in time.

\section{Model Selection}

The simplest model is the one-compartment model with a central compartment. More complicated are multicompartment models where kinetic rates of the exchange between the compartments have to be estimated from the available data. Using the mass balance principle, linear kinetic equations are established as ordinary differential equations. The 
complexity of the multicompartment models and the resulting system of differential equations depends on the identifiability of the model parameters. Sufficient concentration data must be available for each subject in order to solve his/her kinetic equations.

\section{Obtaining Initial Estimates}

The numerical solution of the system equations of a compartment model (i.e. the solution of the system of differential equations) requires the definition of starting values of the kinetic parameters to be statistically estimated iteratively. The stripping method or the peeling of exponential functions is useful to define a set of initial values. Initial estimates are particularly important when data have a lot of scatter or do not span a large enough range of time points to define a full curve. If there are several local minima in addition to an absolute minimum, poor initial values may result in convergence to an unwanted point and hence lead to parameter estimates that are implausible.

\section{Selection of Minimization Algorithm}

It is the goal of the curve fitting procedure to minimize the differences between the observed and predicted concentrations. The most commonly employed criteria are ordinary least squares (OLS), weighted least squares (WLS), and extended least squares (ELS). Among the numerical approaches to the minimization of the objective function approximate solutions have been used. The Downhill Simplex or Nelder-Mead algorithm is based on the geometry of the parameter space and the Powell method on directed one-dimensional minimization. Gradient methods, e.g. the Gauss-Newton or the Levenberg-Marquardt method, require the first derivatives. Second derivatives are used by quasi Newton type methods. Avoidance of local minima is tempted by the use of varying initial values, parameter perturbation (genetic algorithms) or simulated annealing.

\section{Choice of Weights}

Weighting schemes are applied to account for heterogeneity in the variance of the data, particularly if the data span a few or several orders of magnitude. In these instances weights should be assigned to the observations which result in parameter estimates that enable the model to fit low values as well as high values. For many data sets it is appropriate to weight each observation by the reciprocal of the predicted value, or by the square of the predicted value. If weighting is applied, the weighted residuals should be comparable for all data included in the analysis.

\section{Assessing Goodness-of-Fit}

The agreement between model and data (goodness-of-fit) must be checked by diagnostic plots. It is usually evaluated by analyzing the residuals between observed and predicted concentrations. The residuals should be randomly distribu- ted around the predicted curve. Residual plots (residual versus time and residual versus concentration) should be used as diagnostic tools.

\section{Discrimination between Models}

For discrimination between models the F-test, the Akaike Information Criterion (AIC), and the Schwarz Criterion (SC) can be used. The probability that additional parameters are without effect on the sum of least squares is defined by an F distribution. The AIC penalizes the sum of squares by the number of parameters. The SC penalizes the sum of squares by the number of parameters multiplied by the square root of the number of observations. Influence of single measurements and single individuals can be investigated by importance sampling and a sensitivity analysis.

\section{Physiologically Based Pharmacokinetic Models}

Physiologically based pharmacokinetic models (PBPK models) define drug kinetics in terms of the physiology, anatomy and biochemistry of the organism and are composed of compartments which represent body organs and tissues. Further assumptions concern drug uptake, clearance, and allometric scaling. The body compartments are linked together by a flow network. A PBPK model is defined by a system of deterministic kinetic equations (mass balance equations) of the amount or the concentration of the drug in the compartments as a function of time and initial dose. PBPK models are more complex than compartment models and they usually involve a larger number of parameters.

\section{Pharmacokinetic/Pharmacodynamic Modeling}

PK studies the time-dependent fate of a drug and its breakdown products after administration. Pharmacodynamics (PD) is the study of biological effects induced by the drug's effective amount or concentration. Observed PK and PD can be linked by PK/PD modeling. The resulting $\mathrm{PK} / \mathrm{PD}$ models allow the description of the complete time course of the desired and/or undesired effects in response to a drug therapy. When reliable and repeated measurement of the individual PD is possible this approach should be applied in preclinical and clinical trials.

\section{Population Pharmacokinetic Data Analysis}

\section{Standard Two-Stage Approach}

The standard two-stage stage approach (STS) refers to fitting a pharmacokinetic model to the data of each individual. Afterwards summary statistics are computed for the 
total collection of individual parameter estimates. Using this approach, the interindividual variance tends to be overestimated. Moreover, it is not applicable when the individual data are too sparse for individual model fits.

Nonlinear Mixed-Effects Modeling Approach (Population Modeling; see «SOP 14: Population Pharmacokinetic Analysis»)

With this type of modeling not only pharmacokinetic parameters but also interindividual variance parameters are estimated. Mixed-effects or population models are more appropriate than the STS for the analysis of interindividual variation in PK. The parameters of a population model are population means, shift parameters, and interindividual and residual variance parameters.

A population pharmacokinetic data analysis should include relevant covariates, e.g. age, weight, gender, creatinine clearance, co-medication, and concomitant diseases. Quantitative relationships between covariates and pharmacokinetic parameters often help predict individual PK before any individual data have been obtained. The modeling of covariate relationships is of special interest in drug development in order to identify subgroups which may need specific dosage recommendations.

\section{Pharmacokinetic Report}

Pharmacokinetic data should be summarized in a report which should at least include information on the:

- Characteristics of the subjects included in the study,

- Dosage regimen and the drug formulation administered,

- Sample collection, processing and transport to the laboratory,
- Method used for drug analysis and its validation (see 'SOP 12: Validation of Bioanalytical Methods'),

- Software, including the main mathematical equations, used for pharmacokinetic data analysis,

- Special procedures or criteria used during data analysis (e.g. model definition, initial value settings, minimization algorithm, weighting, model discrimination methods, model diagnostics),

- Methods and software used for statistical analysis,

- Tables with individual pharmacokinetic parameters and raw data, if appropriate, and

- An appropriate graphical presentation of the data.

Plots of observed values and model predictions are standard, but should not be restricted to the mean concentration curves. These plots should be supplemented by both raw and weighted residual plots.

Preferably, an integrated report should be prepared that correlates clinical findings and pharmacokinetic parameters. For logistic considerations, such an integrated report may contain a separately prepared pharmacokinetic report as an appendix. As a minimum, clinical and pharmacokinetic reports should cross-reference each other.

\section{References}

Derendorf H, Lesko LJ, Chaikin P, et al: Pharmacokinetic/pharmacodynamic modeling in drug research and development. J Clin Pharmacol 2000;40:1399-1418.

Gabrielsson J, Weiner D: Pharmacokinetic and Pharmacodynamic Data Analysis: Concepts and Applications, 3. ed. Stockholm, Swedish Pharmaceutical Press, 2000.

U.S. Department of Health and Human Services, FDA, CDER and CBER: Guidance for Industry: Population Pharmacokinetics, 1999.

Yacobi A, Skelly JP, Shah VP, Benet LZ: Integration of Pharmacokinetics, Pharmacokinetics and Toxicokinetics in Rational Drug Development. New York, Plenum Publishing Corporation, 1993. 